\title{
AUditive EVIDENZ. ZUM SINNLICHEN VERSTEHEN IN DER MUSIK
}

\section{Dirk Stederoth}

\author{
»Manches, was ich schreibe, verstehe ich ja selber nicht « \\ (Karlheinz Stockhausen in Adorno/Stockhausen 1960: ab 15:18).
}

Das Zitat Stockhausens aus einem Radiogespräch mit Theodor W. Adorno aus dem Jahre 1960 enthält scheinbar eine Paradoxie, die mit dem Verstehen nicht nur von Neuer Musik im engsten Zusammenhang steht. Es ist der scheinbar paradoxe Sachverhalt, dass sich im Felde der Neuen Musik Kompositionen finden, die nicht nur dem Verstehen des Hörers sich entziehen, sondern dem des Komponisten in gleicher Weise, wobei beide (und letzterer insbesondere) nicht davon ausgehen, dass hier ein völlig Irrationales, Willkürliches oder Zufälliges die Komposition geleitet hat. Das Paradoxe dieser Aussage setzt sich dann darin fort, dass es entsprechend im Felde der Neuen Musik möglich ist zu komponieren, ohne vom Verstand im engeren Sinne geleitet zu sein und mithin der Kompositionsprozess von einer Sinnhaftigkeit geleitet wird, die eben gerade nicht in einem Verstehen aufgeht, sondern von einer anderen Beschaffenheit ist, ohne jedoch die sinnhafte Sicherheit zu verlieren, die sonst nur durch den regelgeleiteten musikalischen Verstand gewährleistet wird.

Entsprechend konstatiert Stockhausen im weiteren Verlauf des Gesprächs, es

"gäbe auch eine Art von Verstehen, die sich unter Umständen darin äußert, dass der Mann sagt: >Ich habe nichts verstanden von dem, was sie da gesagt haben . Damit meine ich, dass etwas ins Unterbewusstsein geht und dort arbeitet. Das kann sowohl gefährlich sein, als sich irgendwann für den Mann als sehr nützlich erweisen - Sie wissen, wenn man heute über die Straße geht, wird man von Litfaßsäulen viel mehr beeinflusst, ohne dass man überhaupt weiß, dass man sie gesehen hat, und irgendwann tut man etwas, kauft irgendetwas, und legt sich gar keine Rechenschaft darüber ab, wieso. Aber in einem sehr viel differenzierteren Bereich passiert das ja auch: Wenn ich einer 
Musik ausgesetzt bin, da gibt es ein - es klingt ganz paradox - ein unterbewusstes Verstehen« (Stockhausen, ebd.: ab 25:32).

Stockhausens Hinweis auf ein »unterbewusstes Verstehen«, das von ganz anderer Art ist als das musikalische Verstehen, das sich an musikalischen Regeln und der Form ihrer Anwendung in Kompositionen orientiert, löst somit die scheinbare Paradoxie auf, die in dem Eingangszitat zu liegen schien, insofern nun zwei differente Begriffe des Verstehens vorliegen, wobei derjenige, der im Zitat gebraucht wurde, das regelhafte, bewusste Verstehen betrifft, wohingegen das geschriebene Werk, dem dieses Verstehen verwehrt bleibt, von einer anderen, »unterbewussten« Form des Verstehens geleitet wurde und entsprechend - wenn überhaupt - auch nur einem solchen Verstehen zugänglich ist. Es sei dahingestellt, ob der Begriff des »Unterbewussten« sowie der erläuternde Hinweis auf Priming-Phänomene aus dem Bereich der Werbung hier wirklich den Wink in die richtige Richtung geben, um das Phänomen einer Form des Verstehens jenseits des gewöhnlichen, regelgeleiteten zu illustrieren - auf das Phänomen selbst hingewiesen zu haben, ist Anlass genug, Stockhausen hier zu Wort kommen zu lassen, will sich der folgende Text doch genau diesem Phänomen stellen. Hierbei wird zunächst ganz allgemein aus rezeptionsästhetischer wie produktionsästhetischer Perspektive ein Begriff »auditiver Evidenz « herausgearbeitet, dessen spezifisch popmusikalischen Ausprägungen dann im Anschluss thematisiert werden.

Interessanterweise greift Hans Heinrich Eggebrecht (1995) 35 Jahre nach Stockhausens Gespräch mit Adorno diesen Faden in seinem Buch Musik verstehen wieder auf, wenn er für die Musik-Rezeption zwei Weisen des musikalischen Verstehens unterscheidet: das erkennende und das ästhetische Verstehen. Während er unter »erkennendem Verstehen« die Form eines regelgeleiteten, begrifflichen und sprachlich formulierbaren Verstehens begreift, das die in den musikalischen Regeln festgesetzten Sinnformen bewusst zum Ausdruck zu bringen bestrebt ist, sei das »ästhetische Verstehen « »in seiner reinen Form das im Bereich des Sinnlichen sich abspielende begriffslose Verstehen des in der musikalischen Formung begriffslos objektivierten Sinns der Musik« (ebd.: 35). Dieses Verstehen sei zwar begriffslos und vollziehe sich im Sinnlichen, jedoch folge diesem im Ausgang von dem sinninhärierenden musikalischen Objekt gleichermaßen »adäquates, dem Sinn der Musik angemessenes « (ebd.: 42). Verstehen, insofern in jede tonale Komposition ein »präkompositorisches musikalisches Normen- und Regelsystem « (ebd.: 83) eingegangen sei, das dem Rezipienten in seiner von diesem System geprägten ästhetischen Erfahrung gleichsam aufscheint. Das tonale musikalische Objekt bzw. die tonale Komposition ist also selbst von 
den musikalischen Regeln und Normen solchermaßen durchzogen, dass die sinnliche Erfahrung des von diesen Regeln geprägten Rezipienten ein adäquates Vernehmen des musikalischen Sinns möglich wird, ohne dass dieses Vernehmen bereits bewusste, begrifflich eingeholte und sprachlich ausdrückbare Formen annehmen muss.

Aber nicht nur das allgemeine System von Normen und Regeln wird dabei als musikalischer Sinn erfasst, sondern zugleich die für das einzelne Werk spezifische Ausformung und Ausführung dieser Regeln sowie auch die jeweilige Bestimmung von besonderen, nur für dieses Werk einschlägigen Spielregeln:

»In dem vorkompositorisch existenten und kompositorisch präsentierten Netz des musikalisch Geltenden spielt die Musik ihr Spiel, indem sie das System zur einmaligen Gestalt konkretisiert und individualisiert und dabei auf der Basis des in ihr erscheinenden Regelsystems auch je eigene Spielregeln aufstellen und durchführen kann, die als Definitionsprozesse ad hoc zu verstehen geben und verstanden werden können« (ebd.: 84).

Insofern ist für Eggebrecht im Rahmen des tonalen Systems auch ein adäquates Verstehen des allgemeinen und individuellen Sinns eines musikalischen Werkes auf der Ebene des rein Sinnlichen möglich.

Problematisch wird dieses Modell eines ästhetischen Verstehens jedoch bei der Neuen Musik, die im Verlauf des 20. Jahrhunderts dem »Netz des musikalisch Geltenden « eine gründliche Absage erteilt hat, insofern es mit den klassischen tonalen Regeln und Normen bricht und sich von einem tonalen Regelsystem befreit. Auch wenn diese Befreiung zumindest in der Zweiten Wiener Schule für Eggebrecht nicht vollständig ist, sondern Restbestände des alten Systems weiter tradiert (vgl. ebd.: 88-95), tritt für ihn mit der atonalen Musik eine neue Komponente in das ästhetische Verstehen hinzu, die er in Bezug auf das tonale System von diesem Verstehen differenzierte: der Emotionsgehalt. Aufgrund der subjektiv sich sehr unterschiedlich gestaltenden Reaktionen auf musikalische Werke sei der Emotionsgehalt auch in doppelter Hinsicht von dem ästhetischen Verstehen zu unterscheiden, insofern die Emotionen einmal vom Subjekt und nicht, wie beim ästhetischen Verstehen, vom Objekt ausgingen, und zudem bei den emotionalen Gehalten keine dem ästhetischen Verstehen vergleichbare Adäquation stattfinde, da das sinnliche Subjekt in Bezug auf Emotionen direkt auf sich selbst und eben nicht wie das ästhetische Verstehen auf adäquates Erfassen eines objektivierten Sinns gerichtet sei (vgl. edd.: 72ff.).

Im ästhetischen Erfahren der Neuen Musik nun wird für Eggebrecht diese Differenz überwunden, weil für die Neue Musik eine Einheit von Form und 
Expression einschlägig sei, die auch eine »neue Art des Hörens und Verstehens« (ebd.: 93) erfordere. Bei dieser neuen Art von Verstehen

»sind nun der Formsinn und die Expressionsbedeutung gleichsam miteinander kurzgeschlossen und untrennbar geworden. Der Sinn des Sinns ist es, nicht Formung zu sein, die etwas bedeutet, sondern Bedeutung direkt. Und wenn demnach das ästhetische Verstehen die Expression empfindet, versteht es den Sinn dieser Form, dessen Sinn es ist, Expression zu sein« (ebd.: 95).

Vor diesem Hintergrund lassen sich also mit Eggebrecht zwei unterschiedliche Formen ästhetischen Verstehens differenzieren, wobei die eine Form im Felde der tonalen Musik verortet ist und sich durch ein sinnliches Erfassen der im Objekt aufscheinenden allgemeinen und individuellen Formgehalte auszeichnet, während es sich von den subjektiven Emotionsgehalten getrennt zeigt. Die andere Form, die mit der atonalen Musik einhergeht, trennt sich nicht nur mit dieser von dem tonalen Regelsystem, sondern verwickelt sich zugleich mit den emotionalen subjekthaften Gehalten, da die Formgehalte der atonalen Musik zugleich als Expressionsgehalte zu sehen sind.

Mit diesem Ansatz differenziert Eggebrecht den eingangs geschilderten Anstoß Stockhausens in mehrfacher Hinsicht. Ebenso wie Stockhausen in seiner Rede vom »unterbewussten Verstehen« sowie in seinem Priming-Beispiel geht Eggebrecht von einer nicht-bewussten Prägung aus, die im Rezipienten ein unmittelbar sinnliches, ästhetisches Verstehen ermöglicht, jedoch differenziert er es weiter in ein tonales und atonales ästhetisches Verstehen aus. Weiterhin wird von Eggebrecht durch diese Differenzierung der Grad an Subjektivität und Objektivität eines solchen Verstehens weiter auseinandergelegt, insofern sich für ihn das atonale ästhetische Verstehen gegenüber dem tonalen durch eine stärkere Verwicklung mit dem Rezeptionssubjekt wie auch vermittelt über den »Ausdruckswille[n] des Komponisten« (ebd.: 92) mit dem Produktionssubjekt auszeichnet. Letzteres kommt in Eggebrechts Ansatz leider etwas kurz, geht es ihm doch grundsätzlich zunächst um rezeptionsästhetische Aspekte, wobei in Stockhausens Anstoß, wie das Eingangszitat andeutet, sowohl Rezeptions- als auch Produktionsästhetik im Fokus stand.

Ein jüngerer ästhetischer Ansatz, der die Produzentenperspektive stärker in den Blick bringt, wird von Judith Siegmund (2007) in ihrer Schrift Die Evidenz der Kunst vertreten. Selbst als bildende Künstlerin tätig, stellt sie die Produzentenperspektive in den Mittelpunkt der Analyse, die jedoch so allgemein gehalten ist, dass sie weitgehend problemlos auf die Sphäre der Musik übertragen werden kann. Für sie steht das Kunstwerk in der Mitte 
zwischen dem Produzenten und dem Rezipienten, die jeweils eine unterschiedliche Erfahrungsform dem Kunstwerk gegenüber aufweisen. Die Produzentenperspektive beschreibt sie dabei folgendermaßen:

»In gewisser Hinsicht steht die Künstlerin vor dem Nichts, wenn sie beginnt. Sie geht mit biografischer und gesellschaftlicher Erfahrung um, sie arbeitet in einem vorgegebenen Kontext; aber das Material, in das ihre Erfahrungen transformatorisch hineingearbeitet werden, auch wenn es als ein erschlossenes, als interpretiertes Material vorliegt, wird gerade nicht als eine Gegebenheit von der Künstlerin erlebt, sondern als etwas, das es zu verändern, mindestens zu nutzen gilt, bzw. als etwas, das sich im Prozess verändert. Das bedeutet, dass Künstler ein anderes Verhältnis zum eigenen Werk vor und während seiner Entstehung haben als Rezipienten zum Kunstwerk in der Rezeption« (ebd.: 17).

Das Spezifische der Produzentenperspektive bzw. des Verhältnisses des Künstlers zu seinem herzustellenden Werk ist somit, dass er ein wie auch immer beschaffenes Material vor sich hat, das er allererst durch seinen individuellen Erfahrungskontext, sei's bewusst oder nicht-bewusst, zu modeln hat und in dieser Weise in das Material etwas bzw. sich einbildet, das dem Rezipienten dann als ein »implizites Versprechen« im Kunstwerk entgegentritt:

»Das implizite >Versprechen ‘n die Rezipienten, dass sie im Werk auf fremde Erfahrung treffen können, ist das Fundament dafür, dass Kunstwerke mehr vermögen, als uns lediglich an unsere eigenen Erfahrungen $\mathrm{zu}$ erinnern. Die rezeptionsästhetische Erfahrung von Alterität ereignet sich als Begegnung mit einem bestimmten Anderen« (ebd.: 18, Herv. i. Orig.)

Jenes »Versprechen« kann aber nur eingelöst werden, wenn der Künstler seine Erfahrungen nicht direkt, gleichsam als rein Subjektives in das Kunstwerk einbringt, sondern sie so im Material objektiviert, dass sie einem Rezipienten am Kunstwerk vernehmbar wird. Der Künstler modelt Siegmund zufolge seine eigene Erfahrung also solchermaßen in das Material ein, dass sie als Sinn in dem sinnlichen Material aufgehoben ist und sich dem Rezipienten als solcher Sinn präsentiert, wodurch sich bei diesem eigene Erfahrungsgehalte aktualisieren, jedoch diese zugleich in eine Reibung mit dem im Kunstwerk selbst eingeschriebenen Sinn geraten können. Dieses im Sinnlichen des Kunstwerks durch den Künstler eingearbeitete und somit in jenem aufbewahrte Widerständige ist dann das, was Siegmund als die Evidenz des Kunstwerks versteht, wobei zugleich das Kunstwerk selbst wie auch das, was durch es erfahren werden kann, als evident bezeichnet wird (vgl. ebd.: 127). 
Bezieht man diesen Ansatz nun spezifischer auf den Bereich musikalischer Werke und knüpft an das an, was oben am Ansatz von Eggebrecht herausgearbeitet wurde, so steht ein Begriff im Horizont, den man in Anlehnung an Gottfried Boehms (2008) Begriff der ikonischen Evidenz als auditive Evidenz bezeichnen könnte. Um diesen Begriff mit dem bisher Ausgeführten etwas zu konturieren, sei zunächst danach gefragt, wie sich Siegmunds Evidenzbegriff im spezifisch musikalischen Feld konkretisieren lässt. Demgemäß wäre es der Anspruch des Komponisten, das musikalische Material, das in seiner Beschaffenheit vorliegt, als ein solches zu sehen, in das seine individuelle Erfahrung, kurz: seine Individualität, solchermaßen eingearbeitet wird, dass sie dem Rezipienten als solche erscheinen und er die individuellen Gehalte in der bearbeiteten Form vernehmen kann. Ist dies bei einem musikalischen Werk der Fall, dann kann es nach Siegmund als evident und Evidenzerfahrungen hervorbringendes Werk gelten. Auditive Evidenz läge demnach - ganz allgemein formuliert - dann vor, wenn ein Werk über die formale Beschaffenheit seines Materials hinaus individuelle Gehalte, die der Komponist in es eingearbeitet hat, für einen Rezipienten zum Ausdruck bringen kann.

In dieser Allgemeinheit scheint diese Bestimmung wenig mehr auszusagen als das, was Eggebrecht über die individuelle Ausgestaltung des Normen- und Regelsystems in einer tonalen Komposition ausgeführt hat (s.o.). Zweierlei unterscheidet sie jedoch von Eggebrechts Ansatz. Das ist einmal die Reflexion der Produzentenperspektive und zudem, dass hier nicht zwischen einem, wie man sagen könnte, tonalen und einem emotionalen Ausdruck unterschieden wird. Sie sei deshalb vor diesem Hintergrund nochmals in den Blick genommen. Eggebrecht unterscheidet das ästhetische Verstehen und die emotionale Erfahrung beim Rezipienten ja insbesondere dahingehend, dass dem ästhetischen Verstehen eine Objektivität im Sinne einer Adäquation zukommt, und zwar deshalb, weil im Hörer aufgrund seiner Bekanntschaft mit dem Normen- und Regelsystem der tonalen Musik ein adäquates Erfassen der Form und ihrer individualisierten Ausgestaltung auch im Bereich des Sinnlichen möglich wird. Dagegen sei die emotionale Erfahrung des Rezipienten immer an dessen Subjektivität gebunden und weise entsprechend keine vergleichbare Adäquation auf. Diese saubere Trennung wird jedoch durch die Reflexion der Produzentenperspektive problematisch, scheint Eggebrecht doch davon auszugehen, der Produzent habe im tonalen System die Möglichkeit, seine individuellen Erfahrungen gleichsam verlustfrei in das gegebene Material einzubinden. Nach Siegmund liegt in dieser Einbindung aber bereits eine Reibung zwischen der individuellen Erfahrung des Produzenten und dem Anspruch, diese am Material in eine objektivierte 
Form zu bringen. Die gleiche Spannung findet sich dann aber auch auf der Rezipientenseite wieder, insofern das evidente Werk neben individuellen Erfahrungen auch Reibungspunkte gegenüber diesen hervorbringt. Auf beiden Seiten also liegt demnach vielmehr eine Spannung zwischen Subjektivem und Objektivem vor, die sich beim Produzenten als Widerständigkeit des Materials und beim Rezipienten als Widerständigkeit des Werks gegenüber den jeweiligen subjektiven Erfahrungen zeigt. Und die gleiche Spannung findet sich entsprechend auch im Werk wieder, weil es als Objekt zugleich etwas in sich trägt, das Ausdruck von und Ausdruck für Subjektivität ist.

Vor diesem Hintergrund erscheint dann auch die Trennung, die Eggebrecht zwischen der tonalen und der atonalen Musik hinsichtlich des musikalischen Verstehens vornimmt, in einem neuen Licht. Die Engführung von ästhetischer Erfahrung und Erfahrung von Emotionalität bzw. von Formsinn und Expressivität des Werks, die Eggebrecht für die atonale Musik herausarbeitet, läge somit bereits in der tonalen Musik vor, insofern auch der tonale Komponist in einer Spannung zwischen Subjektivität und Objektivität steht, die gleiches auch beim Rezipienten, wenn auch in anderer Weise aufscheinen lässt. Der Unterschied zwischen tonaler und atonaler Musik läge damit also lediglich in der Unterschiedlichkeit der Beschaffenheit des Materials und der unterschiedlichen Bekanntschaft mit dieser Beschaffenheit beim Rezipienten. Dass diese fehlende Bekanntschaft mit dem Material auch beim atonalen Komponisten ein Verstehen seines Werks behindern kann, was wiederum auf das Eingangszitat von Stockhausen zurückverweist, zeigt das Beispiel Arnold Schönbergs, von dem Adorno in dem Gespräch mit Stockhausen erzählt: dass ihm nämlich eines seiner Werke bis in das späte Alter hinein verschlossen geblieben sei, wobei Adorno darauf verweist, dass es auf der Basis der Bekanntschaft mit dem Material im Jahre 1960 durchaus als verständlich gelten könne. ${ }^{1}$ Dieses Beispiel verweist darauf, dass mit der fortschreitenden Bekanntschaft mit dem Material nicht nur der Grad an Verständnis beim Rezipienten steigt, sondern ebenfalls der Grad des Verständnisses des Komponisten seinem eigenen Werk gegenüber. Adorno geht in

1 »Es wird Sie vielleicht interessieren, Herr Stockhausen, dass diese Äußerung [das Eingangszitat - D.S.], die nun sicherlich die meisten unserer Zuhörer schockieren wird, während sie mir gerade eigentlich [als] etwas außerordentlich Fruchtbares erscheint, dass eine Äußerung ganz analogen Sinnes mir erinnerlich ist von Schönberg, der mir vor zwanzig Jahren einmal in Los Angeles von einem Stück gesagt hat, nämlich von seinem Bläserquintett, er verstehe es selber noch nicht. Nun unterdessen, das ist auch glaube ich interessant, ist gerade dieses Stück für uns verhältnismäßig leicht verständlich geworden und er würde es ohne Frage heute auch verstehen « (Adorno, in: Adorno/Stockhausen 1960: ab 15:23). 
dieser Passage aber noch weiter, indem er in diesen Augenblicken des Nicht-Verstehens des Komponisten seinem eigenen Werk gegenüber gerade die produktiven Momente des Werks sieht, die ein Neues zum Ausdruck brächten, das dem Komponisten selber eben noch nicht bekannt war. ${ }^{2}$ Ohne diesen interessanten Aspekt des musikalisch Neuen hier nun weiter verfolgen zu können, zeigt sich in diesem Aspekt, dass der Materialfortschritt selbst dazu auffordert, dass der Komponist sich in die Spannung zwischen seinen individuellen Erfahrungen, oder - wie Eggebrecht es sagt - seinem Ausdruckswillen und der Notwendigkeit einer Objektivierung im Material begeben muss, wobei noch der zusätzliche Aspekt ins Spiel kommt, dass sich auf diesem Wege auch Erfahrungen des Komponisten in das Werk einspielen, die von ihm selbst als unbewusste Erfahrungen gar nicht als Einbringungen in das Werk intendiert waren. Dieser Aspekt setzt sich natürlich ebenfalls im Rezipienten fort, insofern auch eine Reibung oder Aktualisierungsanlässe des Werks in Bezug auf unbewussten Erfahrungsgehalte beim Rezipienten möglich sind, die auch dazu beitragen können, dass ihm ein Werk aus unverständlichen Gründen verschlossen bleibt oder gerade deshalb eben sehr entgegenkommt.

Die von Adorno konstatierte Fremdheit des Werks gegenüber dem Komponisten, die sich angesichts einer noch mangelnden Vertrautheit mit der Beschaffenheit des Materials oder auch durch unbewusste Einspielungen auszeichnet, gilt zwar im Fortschritt der Beschaffenheit des Materials insbesondere für die Phasen, in denen größere Veränderungen in dieser zu verzeichnen sind (wie das bei Schönberg ja der Fall war), jedoch ist dies angesichts des Fortschreitens der Materialentwicklung nur graduell zu sehen, weshalb eine solche Fremdheit als ebenso grundsätzlich bezogen auf die Materialbeschaffenheit wie auch auf die Beteiligung unbewusster Erfahrungen auf den Kompositionsprozess beurteilt werden muss. Gleiches gilt dann vice versa, wenn auch in etwas anderer Form, für den Rezipienten. Somit lässt

2 »Nun das zeigt, dass das, was Sie gesagt haben, nicht dieses Harmlose meint, dass etwa der sogenannte Augenblick der Inspiration irrational sei und dass man seiner selbst nicht mächtig ist, sondern dass hier schon das gemeint ist, dass die Werke selber gegenüber dem Autor eine Art von Selbstständigkeit, eine Art Eigengesetzlichkeit annehmen können, die es ihm verwehrt, sie in all seinen [ihren] Eigentümlichkeiten sofort und ganz mitzuvollziehen, dass also das Werk seinem eigenen Autor gegenüber auch in einem wirklich radikalen Sinn sich zu entfremden vermag und dass es durchaus möglich ist, dass gerade diese Augenblicke, in denen er am wenigsten das Werk selber versteht, die ihm also die fremdesten sind, dass das die eigentlich produktiven sind, nämlich die, in denen das Neue erscheint, das was er also hervorbringt, ohne dass er es selber bereits gewusst hätte« (Adorno, in: ebd.: ab 16:07). 
sich die von Eggebrecht dargelegte Trennung zwischen tonaler und atonaler Musk hinsichtlich des ästhetischen Verstehens nicht aufrechterhalten, womit die übergreifende Bestimmung der auditiven Evidenz, wie sie oben vorgenommen wurde, von dieser Seite her als gerechtfertigt bezeichnet werden kann.

Doch was bedeutet nun ein solcher Begriff der »Auditiven Evidenz « im Rahmen populärer Musik? Lassen sich hier besondere Merkmale gegenüber der Tradition der sog. Kunstmusik, die ja im bisherigen Verlauf dieses Textes im Vordergrund stand, herausstellen? Um diese Fragen in den Blick zu nehmen, sei zunächst darauf verwiesen, dass die bisherigen Erörterungen sich auf das klassische Modell eines musikalischen Werkes richteten, das von einem Komponisten in einer bestimmten Form festgehalten wird (Partitur oder Ähnliches), damit es dann von Musikern interpretiert einem Publikum präsentiert werden kann. Dieses Modell ist jedoch, wie Daniel Martin Feige darlegt, in Bezug auf den Jazz und hier insbesondere das Spielen von Jazz-Standards problematisch, da beim Standard-Jazz fast alle wesentlichen strukturalen Parameter in der konkreten Performance verhandelbar bleiben und insofern die Musik und ihre konkrete Struktur erst in der Realisierung entsteht (vgl. Feige 2014 sowie Feiges Beitrag in diesem Band). Feige hält daher auch für solche realisierungsorientierte Musik den Werkbegriff letztlich für obsolet bzw. erweitert ihn dahingehend, dass erst die Geschichte der Interpretationen das Werk vervollständige (ebd.: 84f.), worauf hier jedoch nicht näher eingegangen werden kann.

Diesen Gedanken weiterführend hat der Autor des vorliegenden Textes an anderer Stelle (Stederoth 2017) ein grundsätzliches musikästhetisches Spannungsfeld zwischen strukturorientierter Musik, die sich auf festgeschriebene Werke bezieht, und realisierungsorientierter Musik, deren Entstehen an den konkreten Vollzug gebunden ist, dargelegt. Hierbei zählt der Autor zu den realisierungsorientierten Musiken neben dem Standard-Jazz und kunstmusikalischen Ansätzen, die ihre Ausgestaltung an die konkrete Realisierung durch die Musiker binden (beispielsweise Stockhausens Textkompositionen aus den 1960er Jahren), auch weite Teile der populären Musik, bei denen die vorgegebene und auf einem Leadsheet zusammenfassbare tonale, harmonische und rhythmische Struktur ebenso rudimentär ist wie etwa beim Standard-Jazz, auch wenn gegenüber diesem diese Strukturparameter in populärer Musik während der Performance weit weniger verhandelbar sind. Ohne das hier vertiefen zu wollen, wird es jedoch sinnvoll sein, 
den oben erörterten Begriff der auditiven Evidenz auf die besondere Situation realisierungsorientierter Musik hin zu befragen. Da sich die Konsequenzen immer besser am Extrem ablesen lassen, soll entsprechend zunächst die Jazz-Performance als Beispiel dienen, um zu klären, was auditive Evidenz im Rahmen einer Performance bedeuten kann.

In der Regel wird eine Standard-Jazz-Performance bekanntlich in einer Gruppe gespielt, ${ }^{3}$ wobei nach dem gemeinsamen Spiel des Themas (head) abwechselnd Soloimprovisationen folgen, bis das Stück mit dem erneuten gemeinsamen Spielen des Themas endet. Bei dieser Standardform der Standard-Jazz-Performance scheint es auf den ersten Blick so, als würde während einer Solo-Passage nur der Solist improvisieren und die restlichen Musiker lediglich eine standardisierte Begleitung spielen, jedoch ist dies keineswegs der Fall, insofern auch die jeweils begleitenden Musiker improvisierte bzw. spontane rhythmische Akzente, tonale Variationen und harmonische Umstellungen und Erweiterungen vornehmen. In einer StandardJazz-Performance sind somit alle Musiker an der aktualen und spontanen Entstehung, Formung und Ausgestaltung beteiligt, wobei die Lebendigkeit dieser Ausgestaltung davon abhängt, inwieweit die beteiligten Musiker offen sind für die von den jeweils anderen Musikern geäußerten spontanen Ideen, die es produktiv und kreativ in das interpersonale Spiel zurückzugeben gilt. Durch dieses rezeptive und kreativ-produktive Aufeinanderbezogensein entsteht zwischen den beteiligten Musikern eine Objektivität, die einem Organismus gleicht, in dem alle Organe in der ihnen eigenen Weise das Ganze am Leben erhalten. Jeder Musiker ist so Rezipient, Produzent und in einem gewissen Sinne Teil des »Werkes« selbst, das durch die Spontaneität aller Beteiligten aktual als ein dichtes Gewebe von Aktionen und Reaktionen, die jeweils aus objektiven materialen und subjektiv emotionalen Aspekten bestehen, entsteht.

Bezieht man diese Situation auf den oben erörterten Begriff auditiver Evidenz, so lässt sich die Struktur, dass der Komponist seine subjektiven Erfahrungen in einem gegebenen Material verobjektiviert, zunächst auf alle beteiligten Musiker einer Jazz-Performance anwenden, denn alle sind im Aktual der Performance kompositorisch tätig und bringen ihre Subjektivität solchermaßen in das Material ein, dass sie für die jeweils anderen vernehmbar ist. Zugleich aber sind die Musiker in der Performance Rezipienten der spontanen Einwürfe der jeweils anderen Musiker, denen sie mit ihrer Subjektivität, sei's bestärkend, sei's mit Reibung, aktual begegnen. Aus diesem

3 Auf den seltenen Sonderfall einer Einzelperformance sei hier aus Platzgründen nicht näher eingegangen. 
produktiven Wechselspiel auditiver Evidenzen entsteht dann ein musikalisches Ganzes, dem ebenfalls auf einer höheren Ebene Evidenz zukommen kann, wobei diese davon abhängt, inwieweit die gemeinsame Bildung einer Objektivität aus dem Zusammenspiel der einzelnen Musiker glückt, weniger glückt oder gar misslingt. Auch diese auditive Evidenz zweiter Stufe, wie sie genannt werden könnte, kann von den einzelnen Musikern vernommen werden, jedoch zielt sie eigentlich auf das Publikum als Rezipienten. Zwar kann dieses auch seine Aufmerksamkeit auf das Wirken der einzelnen Musiker und damit auf die Evidenzen erster Stufe richten, jedoch liegt sein Hauptohrenmerk zumeist auf dem Vernehmen der Evidenz der Gesamtsituation, die sich in der Performance ereignet und die sich der individuellen Erfahrung des Publikums gegenüber aktualisierend oder widerständig oder beides zugleich zeigen kann. Dass natürlich auch hier alle dargelegten Bestandteile und Ebenen mit dem oben erörterten Aspekt des Unbewussten ergänzt werden müssten, versteht sich von selbst und braucht deshalb nicht eigens ausgeführt werden.

Nun scheint auf den ersten Blick eine solche Jazz-Performance nicht weit von dem Ideal eines Kammerorchesters entfernt zu sein, wie es Adorno formuliert:

»Der erste Schritt, Kammermusik richtig zu spielen, ist, zu lernen, nicht sich aufzuspielen, sondern zurückzutreten. Das Ganze konstituiert sich nicht durch die auftrumpfende Selbstbehauptung der einzelnen Stimmen - sie ergäbe ein barbarisches Chaos - sondern durch einschränkende Selbstreflexion. [...] Große Kammermusikspieler, die im Geheimnis der Gattung sind, neigen dazu, so sehr auf den anderen zu hören, daß sie den eigenen Part nur markieren « (Adorno 1975: 109f.).

Auch im Kammerorchester müssen sich die Musiker also solchermaßen aufeinander beziehen, dass sie ein gemeinsames Ganzes hervorbringen, weshalb die Frage im Raum steht, ob die Unterschiede zwischen einer strukturorientierten und einer realisierungsorientierten Perspektive sich nicht dann auflösen, wenn bei ersterer nicht nur der Komponist und der Rezipient, sondern zudem der Interpret mit in den Fokus rückt. Doch drängt sich sofort ein gewichtiger Unterschied zwischen den Musikern in einem Kammerorchester und denen in einer Jazz-Band auf, dass nämlich jene ihr Spiel an einer fest vorgegebenen Partitur orientieren, während diese keine solche Partitur vorliegen haben, sondern das Stück bis zu einem gewissen Grade erst in der konkreten Realisierung komponieren. Der Interpret einer Partitur greift zwar durch die Generierung der Klangfarbe und die entsprechende Phrasierung in die Präsentation des Materials mit ein und hat entsprechend auch 
einen Anteil an der Realisierung auditiver Evidenz, jedoch bei weitem nicht in dem Maße, wie dies bei Musikern in einer Jazz-Performance der Fall ist. Auch wenn der Interpret nicht in der Radikalität an die Komposition gebunden sein muss, dass in der Klangfarbe jedes Tones die jeweilige Funktion desselben im Werkganzen sich ausdrücken muss, wie es etwa Adorno (2014: 527) fordert, sondern im konkreten Spiel weit mehr Entscheidungen treffen muss, als es Adorno wahrhaben wollte (vgl. Klein 2017), ist der Anteil des einzelnen Musikers an der Komposition selbst eben lediglich auf farbliche und artikulative bzw. phrasierende Momente beschränkt.

Nun ließe sich hier einwenden, dass dies zwar für den engen Kreis einer Standard-Jazz-Performance zutreffen könne, der Musiker einer Mainstreampop-Band dagegen jedoch fast ebenso wenig Spielräume in seiner Performance habe wie der Musiker eines Kammerorchesters. Dieser Einwand trifft insofern zu, als die kreativen Möglichkeiten im aktualen Spiel einer Mainstreampop-Performance weit kleiner sind als bei einer Standard-JazzPerformance. Gleichwohl ist die Variabilität in der Gestaltung eines Popsongs, der ebenfalls lediglich in einem Leadsheet vorliegt, wiederum fast so groß wie im Jazz, wenngleich die Variablen bereits im Vorfeld der Performance festgelegt werden. Die Mainstreampop-Musiker sind insofern auch kompositorisch tätig und etablieren dabei auditive Evidenz im spezifischen Arrangement des Songs, jedoch verbleibt ihnen dadurch weniger kompositorische Freiheit im aktualen Performen des Songs.

Gegenüber dem Jazz wie auch dem Kammerorchester gewinnt in einer Mainstreampop-Performance allerdings der Sound eine große Bedeutung, die inn zu einer der zentralen ästhetischen Kategorien populärer Musik macht (vgl. hierzu Stederoth 2017 sowie die Beiträge in Phleps/v. Appen 2003). Soundgestaltung ist nicht nur ein wesentliches Element im Arrangement eines Songs, wodurch dieser und die Band, die inn spielt, einen individuellen Charakter bekommt, sondern Soundgestaltung ist in einer MainstreampopPerformance auch die Basis dafür, dass die einzelnen Stimmen und Klänge sich gegenseitig zu einem Ganzen fügen können und sich nicht gegenseitig auslöschen oder stören. Damit die Soundidee des Arrangements in einer Performance für einen Rezipienten vernehmbar wird, also ein evidenter Sound und nicht nur ein Brei wechselseitiger Auslöschungen oder Überlagerungen hervortritt, bedarf es eben einer klaren Soundgestaltung, die jene Vernehmbarkeit gewährleistet. Dieses Vernehmen auf der Rezipientenseite kann nun ebenfalls zu einer Aktualisierung von individuellen Erfahrungen führen und somit positiv oder negativ ansprechen oder es kann sich der Sound diesen Erfahrungen gegenüber widerständig zeigen und so einen ei- 
genwilligen Charakter annehmen. Insofern findet sich auditive Evidenz also auch im Rahmen der Soundgestaltung.

Doch nicht nur der Sound spielt in einer Mainstreampop-Performance eine wichtige Rolle, sondern einem weiteren Faktor kommt eine besondere Bedeutung zu, die in einer Jazz-Performance und schon gar in einem Kammerkonzert bei weitem nicht diesen Stellenwert hat: das Publikum. Ist dies in einem Kammerkonzert meist in der Rolle des passiven Rezipienten (was bei einer Jazz-Performance häufig ebenso der Fall ist), wird das Publikum im Rahmen einer Mainstreampop-Performance mehr oder weniger zu einem Akteur in dem Geschehen, da es sich durch Mitsingen, Mitklatschen, Tanzen etc. aktiv in das Gelingen der Performance einbringt. Diese Aktivität gestaltet aber nicht nur die Atmosphäre der Performance, sondern wirkt direkt auf die Musiker und ihre Performance zurück und hat damit - verglichen mit einem Symphoniekonzert - mehr die Rolle eines Dirigenten denn die eines passiven Rezipienten inne. Entsprechend entscheidet sich die Güte einer Mainstreampop-Performance insbesondere auch daran, inwiefern die Musiker fähig sind, diese Aktivität wahrzunehmen und in das eigene Spiel zu integrieren, wodurch dann eine Rückwirkungsschleife entsteht, die sich direkt auf das musikalische Geschehen auswirkt.

Dies verweist auf einen Aspekt, der im bisherigen Verlauf der Darstellung noch viel zu wenig Beachtung gefunden hat, und zwar den Anteil leiblicher Aktivität am musikalischen Geschehen. Stand bisher die auditive Evidenz als ein sinnlich-ästhetisches Phänomen im Mittelpunkt der Erörterung, so haben leibliche Aspekte - wenn überhaupt - nur im Rahmen passiver Emotionen in Eggebrechts Konzept und in Anknüpfung an dieses eine Rolle gespielt. Jedoch ist sinnliches Verstehen in Form des Vernehmens auditiver Evidenz auch auf leiblicher Ebene möglich, was sich insbesondere am Begriff und Phänomen des Groove zeigen lässt. Dass Groove als ein zentraler Bestandteil von Mainstreampop gelten kann, wurde bereits an anderer Stelle dargelegt (Stederoth 2017) und kann hier nicht nochmals ausführlich erörtert werden. Für den hier gestellten Problemkontext ist es allerdings wichtig festzuhalten, dass sich Groove im unmittelbaren Etablieren von Körperbewegungen zeigt, die vom Tippen des Fußes, dem Schwelgen bei einer Ballade bis zum ekstatischen Tanzen reichen können. Stellt sich hingegen keinerlei spontane Bewegung beim Zuhörer einer Pop-Performance ein, so gibt es für dieses Phänomen zunächst nur zwei plausible Erklärungen: Entweder ist die Pop-Band nicht fähig, Groove in der Musik zu etablieren, weshalb beim Hörer auch kein entsprechender Impuls ausgelöst wird, oder aber der Zuhörer spricht nicht auf die spezifische Grooveform an, die ihm darge- 
boten wird. Die erste Erklärung betrifft somit mehr die Produzentenseite und die zweite eher die des Rezipienten.

Was die Produzentenseite betrifft, so sei hier auf ein Zitat des USamerikanischen Jazz-Schlagzeugers Jo Jones verwiesen - sagte er doch einmal recht bündig: »Die beste Art, in der du sagen kannst, was swing ist, ist, daß du ihn spielst, wenn du ihn fühlst, oder du spielst ihn überhaupt nicht« (Jo Jones, zit. n. Berendt 1981: 203f.). Das Geheimnis des Groove wäre demnach nicht, dass man bestimmten Regeln oder Normen folgt, was bei solch minimalen mikrorhythmischen Abweichungen, die in der Musikwissenschaft für das Phänomen Groove verantwortlich gemacht werden (vgl. Gerischer 2010, Pfleiderer 2006, Keil/Feld 1994), auch kaum möglich wäre, sondern dass der Musiker selbst den Groove oder Swing spüren muss, damit er ihn spielen kann. Hier zeigt sich ein sehr deutlicher Unterschied zur Position des Komponisten im obigen Modell: Ein Komponist muss bspw. nicht traurig sein, um eine traurige Passage im Werk evident zu etablieren, und auch ein Interpret muss nicht mit dieser Emotion erfüllt sein, um sie adäquat auf seinem Instrument zu realisieren; dem Pop-Musiker kommt dagegen bei der Etablierung von Groove genau diese Identität mit dem zu etablierenden Gefühl zu, wenn Groove erst dann entstehen kann, wenn die Musiker inn selbst im Spiel fühlen. Dies ist denn auch der Grund dafür, dass tippende Füße oder auch ekstatische Tanzbewegungen in einer Mainstreampop-Performance nicht nur das Geschehen vor einer Bühne, sondern gleichermaßen auf der Bühne kennzeichnen. Vor diesem Hintergrund lässt sich die Etablierung von Groove von Produzentenseite aus ebenfalls als ein Anspruch auf Herstellung von auditiver Evidenz auf einer leiblichen Ebene bestimmen, die vom aktual realisierten Werk direkt auf die Leiblichkeit der Rezipienten ausstrahlt.

Was den Rezipienten betrifft, so bringt dieser natürlich immer seine individuelle leibliche Konstitution mit, in die auch seine individuellen Erfahrungen eingegangen sind. Dieser leibliche Erfahrungsgehalt kann nun durch einen Groove aktualisiert werden und sich demgemäß ebenfalls in tippenden Füßen oder ekstatischen Tänzen äußern, oder aber die spezifische Form von Groove weist zu viel Widerständiges gegenüber den leiblichen Erfahrungsgehalten auf, was dann einer unmittelbaren leiblichen Reaktion im Wege stehen kann. Somit entsprich auch die Rezipientenseite exakt der Struktur des Vernehmens auditiver Evidenz, nur dass sie sich hier in unmittelbar leiblichen Sphären ereignet und entsprechend das ästhetische Verstehen sich in einer leiblichen Regung äußert.

Somit lässt sich abschließend festhalten, dass sich auditive Evidenz nicht nur im Felde strukturorientierter Musik als ein Konzept zum Begreifen sinnli- 
chen Verstehens etablieren lässt, sondern zudem für alle wesentlichen kategorialen Bereiche realisierungsorientierter Musik einschlägig ist, was dieser

Text sich vorgenommen hatte zu zeigen.

\section{Literatur}

Adorno, Theodor W. / Stockhausen, Karlheinz (1960). »Der Widerstand gegen die neue Musik. " Diskussion zwischen Theodor W. Adorno und Karlheinz Stockhausen, aufgenommen im Hessischen Rundfunk, Sendung am 22.4.1960, verfügbar unter: https://archive.org/details/AdornoKarlheinzStockhausen-GespraechUebe rNeueMusik1960.

Adorno, Theodor W. (1975). Einleitung in die Musiksoziologie. Frankfurt/M.: Suhrkamp.

Adorno, Theodor W. (2014). Kranichsteiner Vorlesungen. Nachgelassene Schriften. Abteilung IV: Vorlesungen. Bd. 17. Hg. v. Klaus Reichert u. Michael Schwarz. Berlin: Suhrkamp.

Berendt, Joachim E. (1981). Das große Jazzbuch. Von New Orleans bis Jazz Rock. Frankfurt/M.: Krüger (5., vollst. überarb. u. aktual. Ausg.).

Boehm, Gottfried (2008). »Augenmaß. Zur Genese der ikonischen Evidenz.«In: Movens Bild. Zwischen Evidenz und Affekt. Hg. v. dems., Birgit Mersmann u. Christian Spies. München: Fink, S. 15-43.

Eggebrecht, Hans Heinrich (1995). Musik verstehen, München: Piper.

Feige, Daniel Martin (2014). Philosophie des Jazz. Berlin: Suhrkamp.

Gerischer, Christiane (2010). »Groove - magische Momente - Versuch einer rationalen Annäherung«, in: PopSkriptum 11, https://www2.hu-berlin.de/fpm/ popscrip/themen/pst11/pst11_gerischer.html, Zugriff: 31.7.2018.

Keil, Charles / Feld, Steven (1994). Music Grooves. Chicago: University of Chicago Press.

Klein, Richard (2017). »Adorno als negativer Hermeneutiker. Zu seiner Theorie der musikalischen Interpretation. « In: Zeitschrift für kritische Theorie 44/45, S. 158-175.

Pfleiderer, Martin (2006). Rhythmus. Psychologische, theoretische und stilanalytische Aspekte populärer Musik. Bielefeld: transcript.

Phleps, Thomas / Appen, Ralf von (Hg.) (2003). Pop Sounds. Klangtexturen in der Pop- und Rockmusik. Basics - Stories - Tracks. (= texte zur populären musik 1). Bielefeld: transcript.

Siegmund, Judith (2007). Die Evidenz der Kunst. Künstlerisches Handeln als ästhetische Kommunikation. Bielefeld: transcript.

Stederoth, Dirk (2017). "Sound, Groove, Performance. Musikästhetische Realisierungskategorien zur Charakterisierung populärer Musik. «In: Was ist Popmusik? Konzepte, Kategorien, Kulturen. Hg. v. Timo Hoyer, Carsten Kries u. Dirk Stederoth. Darmstadt: Wissenschaftliche Buchgesellschaft, S. 111-134. 


\begin{abstract}
This text initially develops a general concept of auditive evidence that provides an explanation for how a work of art can express individual contents, incorporated by the composer and beyond the formal nature of its material, to the recipient. For this purpose, it considers both the aesthetic response, specifically Hans Heinrich Eggebrecht's concept of a sensual understanding of music which he conceives of alongside a conceptual understanding that orients itself upon rules of composition as well as the production aesthetics in form of the conceptualized term evidence which Judith Siegmund has developed for the visual arts.

In the second part of the text, this concept is applied to the specific contents of popular music. In doing so, the compositional process of a standard jazz performance is initially focused upon. Furthermore, the concept is substantiated on the basis of the three central popular music categories of sound, groove, and performance.
\end{abstract}

